\title{
Wavelet-Based Method for Time-Domain Noise Analysis and Reduction in a Frequency-Scan Ion Trap Mass Spectrometer
}

\author{
Szu-Wei Chou, ${ }^{1,3}$ Guo-Rung Shiu, ${ }^{1}$ Huan-Cheng Chang, ${ }^{2}$ Wen-Ping Peng ${ }^{1,3}$ \\ ${ }^{1}$ Department of Physics, National Dong Hwa University, Shoufeng, Hualien, Taiwan 97401, Republic of China \\ ${ }^{2}$ Institute of Atomic and Molecular Sciences, Academia Sinica, Taipei, Taiwan 10617, Republic of China \\ ${ }^{3}$ AcroMass Technologies Inc, Taipei, Taiwan 114, Republic of China
}

\begin{abstract}
We adopt an orthogonal wavelet packet decomposition (OWPD) filtering approach to cancel harmonic interference noises arising from an $\mathrm{AC}$ power source in time domain and remove the resulting if voltage interference noise from the mass spectra acquired by using a charge detection frequency-scan quadrupole ion trap mass spectrometer. With the use of a phase lock resampling technique, the transform coefficients of the if interference in signals become a constant, exhibiting a shift of the baseline in different if phases. The if interference is therefore removable by shifting the baselines back to zero in OWPD coefficients. The approach successfully reduces the time-domain background noise from 1367 electrons (rms) to 408 electrons (rms) (an improvement of $70 \%$ ) and removes the high frequency noise components in the charge detection ion trap mass spectrometry. Unlike other smoothing or averaging methods commonly used in the mass-to-charge $(\mathrm{m} / \mathrm{Ze})$ domain, our approach does not cause any distortion of original signals.
\end{abstract}

Key words: Ion trap mass spectrometry, Signal processing, Wavelet packet decomposition, Frequency scan, Noise reduction, Charge detector

\section{Introduction}

$\mathrm{D}$ enoising is an important data processing method in improving the signal-to-noise ratio $(\mathrm{S} / \mathrm{N})$ of a spectrum. Most denoising approaches currently adopted in mass spectrometry are to smooth spectra or average the noises in mass-to-charge $(\mathrm{m} / \mathrm{Ze})$ domain [1-8]. Barclay et al. compared various methods for smoothing in processing spectra, including orthogonal transform (Fourier transform), windowed smoothing algorithms (Savitzky-Golay or Kalman averaging), and discrete wavelet decomposition or discrete

Electronic supplementary material The online version of this article (doi:10.1007/s13361-012-0455-2) contains supplementary material, which is available to authorized users.

Correspondence to: Wen-Ping Peng; e-mail: pengw@mail.ndhu.edu.tw wavelet packet decomposition [9]. They found that the wavelet-based methods result in minimal distortion of original data. $\mathrm{Li}$ et al. later showed that by proper selection of wavelet functions and decomposition levels, the noise embedded in the mass spectrometry data can be substantially removed [6]. However, these denoising approaches did not characterize the modules of noise features, and unavoidably lead to distortion of original signals when they are transformed from the time domain to the $m / Z e$ domain. Hence, there is a desire to develop methods to remove the noises without domain transformation [10]. $\mathrm{Xu}$ proposed to perform distortion-free harmonic interference noise cancellation in the time domain with a wavelet-based method and demonstrated its feasibility by using a simulated piecewise smooth signal and a real and irregularly pulsating signal [11, 12]. The method provides a useful alternative to improve $\mathrm{S} /$ $\mathrm{N}$ ratios. 
For a signal observed in a mass spectrum, it is typically composed of three parts [11-13]:

$$
\mathrm{S}(t)=\mathrm{S}_{\mathrm{x}}(t)+\mathrm{N}_{\mathrm{h}}(t)+\mathrm{N}_{\mathrm{w}}(t)
$$

where $\mathrm{S}(\mathrm{t})$ is the observed signal, $\mathrm{t}$ is the time, $\mathrm{S}_{\mathrm{x}}(\mathrm{t})$ is the original signal without noise interference, $N_{h}(t)$ is the harmonic interference induced by the $\mathrm{AC}$ power source, and $N_{w}(t)$ is the zero-mean Gaussian white noise. The last term is encountered whenever electrons cross the $p n$ interfaces in electronic circuits and can be reduced by using a Boxcar averaging method or low pass filters [14]. Many researches use notch filters and comb-shaped filters [15-21] to remove $\mathrm{N}_{\mathrm{h}}(\mathrm{t})$ but they cause distortion of original signals $[11,12]$. Xu has shown that it is possible to remove $\mathrm{N}_{\mathrm{h}}(\mathrm{t})$ by a harmonic interference cancellation algorithm, which cancels the $\mathrm{N}_{\mathrm{h}}(\mathrm{t})$ completely, reduces $\mathrm{N}_{\mathrm{w}}(\mathrm{t})$ and retains the signal without distortion [11]. In this study, we apply the algorithm to remove the time-domain electronic noises in the ion signals acquired with a home-built laser desorption/ ionization charge detection ion trap mass spectrometer (LDCD-ITMS) [22-27].

In analyzing the mass spectra, we notice that Equation (1) is not enough to describe the signal components because the applied $\mathrm{rf}$ driving voltage for the ion trap is high and will inevitably interfere $\left(\mathrm{N}_{\mathrm{RF}}(\mathrm{t})\right)$ with the signal $(\mathrm{S}(\mathrm{t}))$. We know of no methods reported to remove the $\mathrm{N}_{\mathrm{RF}}(\mathrm{t})$ due to coupling of $S_{x}(t)$ with the $r f$ driving voltage. Here, we present an OWPD method to achieve the task and show that $\mathrm{N}_{\mathrm{RF}}(\mathrm{t})$ is the dominant noise in the mass spectra obtained with LDCD-ITMS [22-29]. The removal of $\mathrm{N}_{\mathrm{RF}}(\mathrm{t})$ is critically important in our application of the LDCD-ITMS technique to measure the masses of nanometer-sized particles, of which higher $\mathrm{rf}$ frequencies are required for the trapping. Using trapping frequencies higher than $1 \mathrm{kHz}$ gives rise to distinct noises superimposed on the signals, as clearly observed in detection of $0.7 \mu \mathrm{m}$ polystyrene spheres and Escherichia coli. Our denoising method successfully removes the noises without any distortion and hence improves the $\mathrm{S} / \mathrm{N}$ ratios.

With the denoising program developed in this work, we are able to reduce substantially the levels of $\mathrm{N}_{\mathrm{h}}(\mathrm{t})$ and $\mathrm{N}_{\mathrm{RF}}(\mathrm{t})$, which makes the further reduction of white noise of the charge detector possible. Currently, the mass resolution of our frequency-scan ion trap mass spectrometer is $\sim 10$. The reduction of electronic white noise will not only improve the resolution of the mass spectrometer but also enhance its detection sensitivity for detecting nanoparticles.

\section{Experimental}

\section{Instrument}

In previous experiments $[24,28]$, we reported the development of a new type of mass spectrometry for rapid measurement of the masses and the mass distributions of cells and polystyrene microparticles by measuring mass-tocharge ratio $(\mathrm{m} / \mathrm{Ze})$ and charge $(Z)$ simultaneously using a charge-sensitive detector. Figure 1a shows a schematic diagram of the experimental setup, in which a 0.4-mm-thick antimony-doped single-crystal silicon (100) wafer was used as the target plate. Samples of microparticles were loaded directly onto the target plate without a matrix and placed in one of the holes on the ring electrode of the quadrupole ion trap (QIT). A Nd:YAG laser beam ( $\lambda=1064 \mathrm{~nm}$, Q-switch) with a pulse duration of approximately $6 \mathrm{~ns}$ was shined directly onto the $\mathrm{Si}(100)$ wafer to desorb particles, which were subsequently captured by the QIT, with a power density of around $10^{7} \mathrm{~W} / \mathrm{cm}^{2}$.

Frequency scan of the QIT was conducted in a monopolar mode with 1000 steps per second for linear stepwise scan [30]. The duration of each step was $1 \mathrm{~ms}$. A function generator (NI PXI 5402) provided the rf signal with a variable frequency at a constant amplitude. The rf voltage was amplified by a power amplifier and applied to the ring electrode. Both the end-cap electrodes were electrically grounded to reduce the noise interference. The trapped charged particles were ejected from the holes of the end-cap electrodes in a mass-selective axial instability mode by scanning the rf frequency. A home-built charge detector measured the absolute number of charges carried by the ejected particles. The charge detector comprised a circuit metal acted as a Faraday plate to collect the image charge induced by ions. The charge-integrator used a low-noise JFET transistor as the input stage and an operational amplifier with a basic low-pass filtering. The gain of the charge integrators was calibrated by applying a known voltage pulse across a $1 \mathrm{pF}$ capacitance to simulate the incoming pulse. Without turning on rf field, the output voltage noise converted to electron number was about 500 $[23,24]$. Metal mesh was placed between the endcap and charge detector to effectively reduce the rf pickup. The ion signal was recorded by data acquisition (DAQ) card (NI PXI 6133, National Instrument, Austin, Texas, USA) and the sampling rate of DAQ card was set at $0.5 \mathrm{MHz}$.

A problem associated with the frequency scan is that the amplitude of the $\mathrm{rf}$ field varied with the frequency during the scan. To solve this problem, the DAQ card also recorded the voltage (after attenuation) applied to the ring electrode to calibrate both the gain margin and phase margin. Figure $1 \mathrm{~b}$ shows a scheme for the synchronization of the data acquisition process. Both the function generator and the DAQ card were synchronized by PCI eXtensions for instrumentation (PXI) module (National Instrument, Austin, Texas, USA).

\section{Test Data}

The electronic calibration for the charge detector was carried out by applying an electrical pulse of known amplitude and shape to the "test pulse input" connector of the detector. We collected the test data under the frequency scan mode while 
(a)

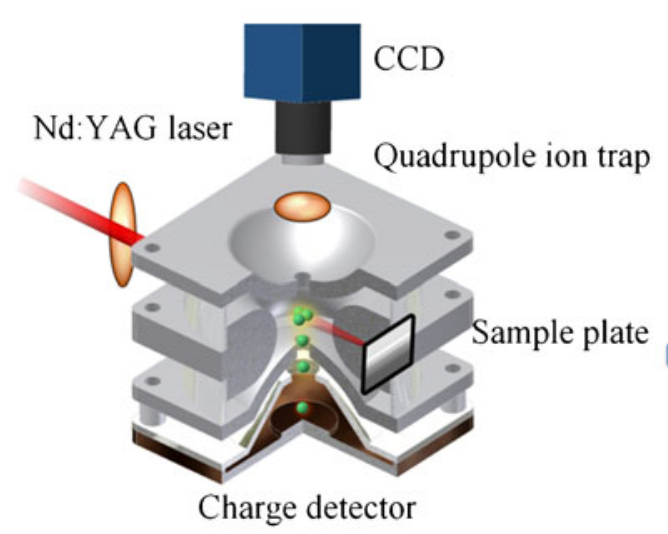

(b)

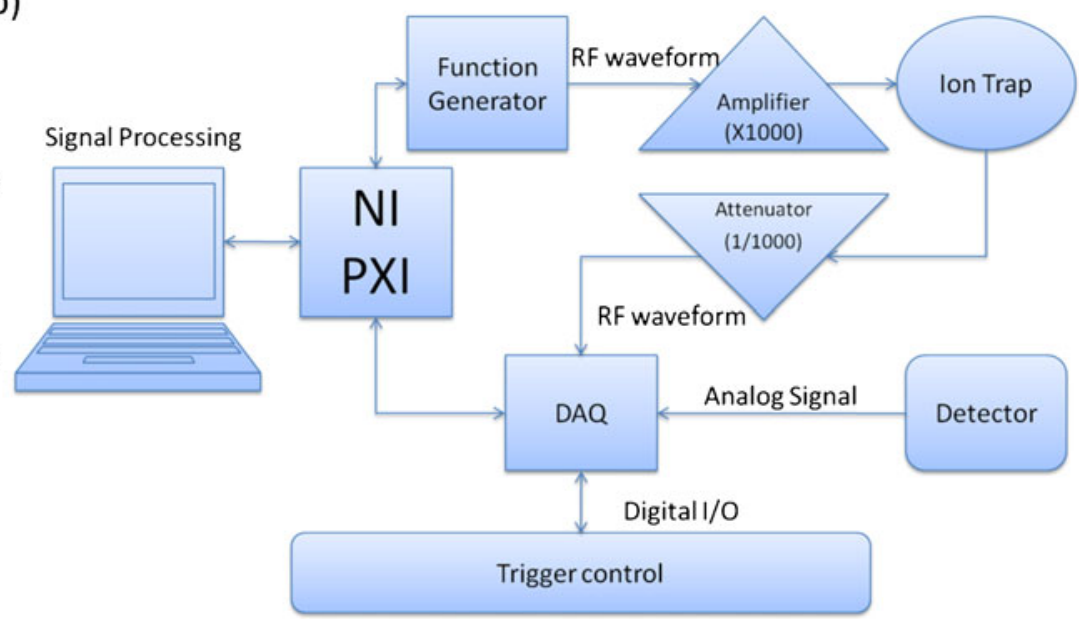

Figure1. (a) Schematic of a laser desorption charge detection ion trap mass spectrometer (LDCD-ITMS). LDCD-ITMS consists of three parts: a laser desorption ion source, a quadrupole ion trap (QIT), and a charge detector. (b) Data acquisition work flow. In LDCD-ITMS, a high voltage power amplifier is used to derive an if waveform from function generator and applied to the ring electrode of QIT. A data acquisition (DAQ) card with two channels records the analog voltage signals from the charge detector and the attenuated if voltage is synchronized with a high rate trigger source. The PCl eXtensions for instrumentation (PXI) module is adopted in data acquisition

the amplitude of the rf voltage was set as $850 \mathrm{~V}_{0-p}$. The test pulse voltage was attenuated by two resistors with a factor of $1 / 100$, and then fed to a $1 \mathrm{pF}$ capacitor; the other terminal of the capacitor was virtually grounded to the amplifier input. The test pulse induced a charge Q in the Faraday plate as follows:

$$
Q=1 p F \frac{V_{\text {test }}}{100}
$$

where $V_{\text {test }}$ is the amplitude of the test pulse.

\section{Real Data}

The real data are signals obtained by using LDCD-ITMS with polystyrene microparticles as the test samples. Polystyrene microparticles with the sizes of $2,4,6,8$, and $9 \mu \mathrm{m}$ in diameter and the Escherichia coli powder were purchased from Sigma-Aldrich (Missouri, USA), polystyrene microparticles with the size of $3.0 \mu \mathrm{m}$ were obtained from the National Institute of Standards and Technology of the United States, and polystyrene microparticles with the size of $0.7 \mu \mathrm{m}$ were obtained from Spherotech (Lake Forest, Illinois, USA). All the polystyrene beads and microorganism were thoroughly washed twice with deionized water and loaded onto the silicon wafer and air-dried.

\section{Algorithm Development}

Both the data were processed and algorithms were written by using LabVIEW (Professional, ver. 8.6 for Windows;
National Instrument). The computer configuration was an Intel Core i5 M450 at $2.4 \mathrm{GHz}$ with $3.2 \mathrm{~GB}$ of RAM.

\section{Modeling Approach}

\section{Observation Model of Signal}

A model to describe the signal of LDCD-ITMS is as follows:

$$
S(t)=S_{x}(t)+N_{h}(t)+N_{R F}(t)+N_{w}(t)
$$

where the signal $\mathrm{S}(\mathrm{t})$ obtained from LDCD-ITMS can be decomposed as a sum of four components: the signal without noises $\mathrm{S}_{\mathrm{x}}(\mathrm{t})$, the harmonic interference induced by the AC power source $\mathrm{N}_{\mathrm{h}}(\mathrm{t})$, rf interference from the applied driving voltage of the ion trap $\mathrm{N}_{\mathrm{RF}}(\mathrm{t})$, and the zero-mean Gaussian white noise $\mathrm{N}_{\mathrm{w}}(\mathrm{t})$. $\mathrm{N}_{\mathrm{w}}(\mathrm{t})$ is random and disordered. Here we concentrate on the discussion of the model of $\mathrm{N}_{h}(\mathrm{t})$ and $\mathrm{N}_{\mathrm{RF}}(\mathrm{t})$.

Figure shown in supporting information $\mathrm{A}$ is the power density distribution of a real signal. We can observe the intensity of harmonic interference in supporting information A. $\mathrm{N}_{\mathrm{h}}(\mathrm{t})$ is described as [11]

$$
N_{h}(t)=\sum_{i=1}^{H} \gamma_{i} \sin \left(2 \pi v_{i} t+\varphi_{i}\right)
$$

$\gamma_{\mathrm{i}}, v_{\mathrm{i}}, \varphi_{\mathrm{i}}$ and are the amplitude, frequency and the initial phase of $i$ th harmonic. $\mathrm{H}$ is the highest order of harmonics, $v_{i}=\mathrm{i} \times v_{1}$, and, where $v_{1}$ is base frequency of the harmonics. Symbol $t$ is time. 
The rf interference $\left(\mathrm{N}_{\mathrm{RF}}(\mathrm{t})\right)$ is induced by applied $\mathrm{rf}$ driving voltage of QIT $\left(\mathrm{V}_{\mathrm{RF}}(\mathrm{t})\right)$, and we assume that $\mathrm{N}_{\mathrm{RF}}(\mathrm{t})$ is linearly proportional to $\mathrm{V}_{\mathrm{RF}}(\mathrm{t})$,

$$
N_{R F}(t)=\varepsilon V_{R F}(t)=\varepsilon A \sin (2 \pi p(t))
$$

where $\varepsilon$ is the coefficient of interference, the value of $\varepsilon$ depends on the rf strength, A is the amplitude of the $\mathrm{rf}$ driving voltage, and $p(t)$ is the phase of driving voltage of QIT. The gain margin of A can be ignored because the effect is negligible in $\mathrm{N}_{\mathrm{RF}}$. Since we generate the frequency scan waveform by phase-continuous frequency hopping, the $p(t)$ is described as

$$
p(t)=f_{\left|\frac{t}{\Delta t}\right|} \times(t \% \Delta t)+\sum_{k=0}^{\left|\frac{t}{\Delta t}\right|-1} f_{k} \Delta t+\frac{\phi_{f_{k}}}{2 \pi}
$$

where $\Delta \mathrm{t}$ is the duration that stays at a certain frequency, $\downarrow\rfloor$ is the round down operator, $f_{k}$ is the frequency at stepwise sweep frequency step $\mathrm{k}, \phi_{f_{k}}$ is the phase shift at $\mathrm{f}_{\mathrm{k}}$ which can be estimated by tone analysis according to the read back value from QIT driving voltage, and \% indicates the modular.

The number of steps is set as $\mathrm{K}$, the frequencies of a linear frequency stepwise scan are described as

$$
f_{k}=\frac{k}{K} \times\left(f_{f}-f_{i}\right)+f_{i}
$$

$f_{i}$ and $f_{f}$ are the initial frequency and the final frequency in a frequency scan.

\section{Noise Rejection}

Using above feature model of noises, a denoising program has been developed to remove $\mathrm{N}_{h}$ and $\mathrm{N}_{\mathrm{RF}}$, and reduce $\mathrm{N}_{\mathrm{w}}$, and the flow chart of the denoising program is shown in supporting information $\mathrm{D}$. The denoising program consists of three major algorithms: Boxcar averaging, harmonic interference cancellation, rf interference reduction. Orthogonal wavelet packet decomposition (OWPD) [31] is the major math tool which we use in denoising algorithm. Detailed OWPD method is discussed in supporting information $\mathrm{B}$.

\section{Boxcar Averaging}

Before we do the OWPD, the size of datasets is reduced by Boxcar averaging to enhance the computing speed and reduce the white noise. The sampling rate of raw data sets after Boxcar averaging should keep four hundred times of the initial frequency during a frequency scan, which can ensure raw datasets have enough resolution to reconstruct the rf interference array.

\section{Harmonic Interference Cancellation}

Harmonic interference cancellation was mentioned by $\mathrm{Xu}$ [11]. We show this noise rejection method in supporting information $\mathrm{C}$.

\section{rf Interference Reduction}

To reduce the rf interference, the duration of scan step is 1 millisecond in linear stepwise frequency scan. The maximum value of phase $\mathrm{p}_{\mathrm{rs}}[\mathrm{m}, \mathrm{n}]$ of a frequency scan is calculated by Equation (6). We create a two-dimension array $\mathrm{p}_{\mathrm{rs}}[\mathrm{m}, \mathrm{n}]$ as

$$
\begin{aligned}
p_{r s}[m, n]=n+\frac{m}{360} \quad & \{m, n\} \in \mathbb{Z}, \\
& 0 \leq m<360, \\
& \text { and } p_{r s}[m, n]<p_{\max }
\end{aligned}
$$

where $m$ and $n$ are indexes of row and column. We calculate the time stamp array $t_{\mathrm{rs}}[\mathrm{m}, \mathrm{n}]$ by doing linear interpolation of Equation (6) using $\mathrm{p}_{\mathrm{rs}}[\mathrm{m}, \mathrm{n}]$. Then we interpolate the signal (S) with timestamp $\mathrm{t}_{\mathrm{rs}}[\mathrm{m}, \mathrm{n}]$, i.e.,

$$
S[m, n]=S\left(t_{r s}[m, n]\right)
$$

where the $\mathrm{rf}$ interference in two-dimension array $\mathrm{S}[\mathrm{m}, \mathrm{n}]$ is as

$$
\begin{aligned}
N_{R F}[m, n]= & \varepsilon A \sin \left(2 \pi p\left(t_{r s}[m, n]\right)\right)=\varepsilon A \sin \left(2 \pi p_{r s}[m, n]\right) \\
& =\varepsilon A \sin \left(2 \pi\left(n+\frac{m}{360}\right)\right)=\varepsilon A \sin \left(2 \pi \frac{m}{360}\right)
\end{aligned}
$$

$\mathrm{N}_{\mathrm{RF}}[\mathrm{m}, \mathrm{n}]$ is a constant in a signal if the phases of the driving voltage are the same. In other words, $\mathrm{S}[\mathrm{m}, \mathrm{n}]$ has a nonzero baseline, which is induced by $\mathrm{N}_{\mathrm{RF}}$, in each row. The resampling signal $\mathrm{S}[\mathrm{m}, \mathrm{n}]$ is shown in Figure 2, where Figure $2 \mathrm{a}$ is the signal after harmonic cancellation. Figure $2 \mathrm{~b}-\mathrm{g}$ are the resampling signals and their baseline fittings are at $0,60,120,180,240$, and $300^{\circ}$. In order to clearly observe the noise characteristics of the charge detector under the influence of strong rf field, we conduct noise measurement with the known test pulses as input and record the output response of charge detector in a frequency scan mode while the driving voltage of QIT is set as $850 \mathrm{~V}$. Figure $3 \mathrm{a}$ shows the noise baseline intensity and the signal induced by $-150 \mathrm{mV}$ test pulse in different phases. We found the shift in measured noise baseline intensity is matched to the measured test pulse signal. This means the rf driving voltage will produce an interference, which is linearly combined with the sinusoidal signal according to Equation (5). Figure $3 \mathrm{~b}$ shows the phase of the ejected particles is at $200^{\circ}$ of the applied rf driving phase. At the phase of $200^{\circ}$, the ejected population is $\sim 25 \%$. This means that $\mathrm{N}_{\mathrm{RF}}$ not only induces a wider noise distribution, but also causes a systematic error in charge measurement. 

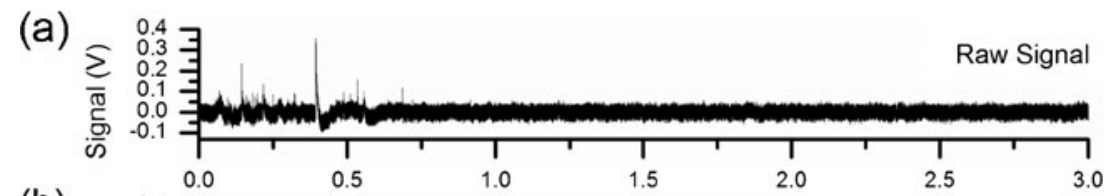

(b)

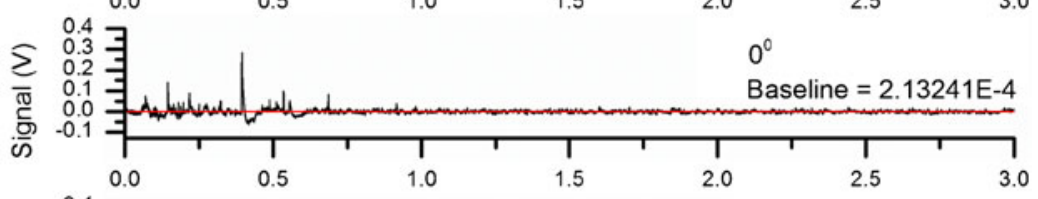

(c)

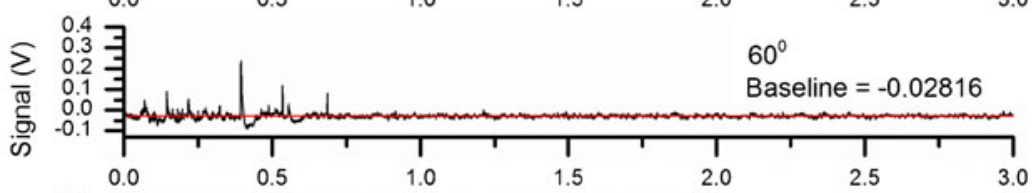

(d)

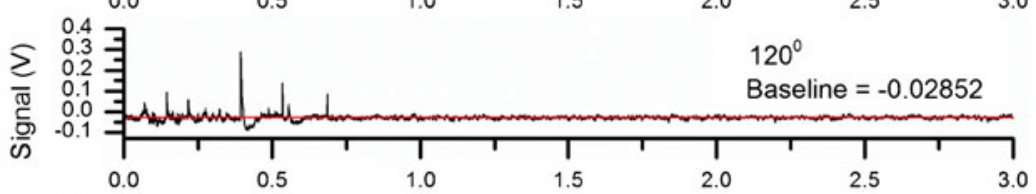

(e)

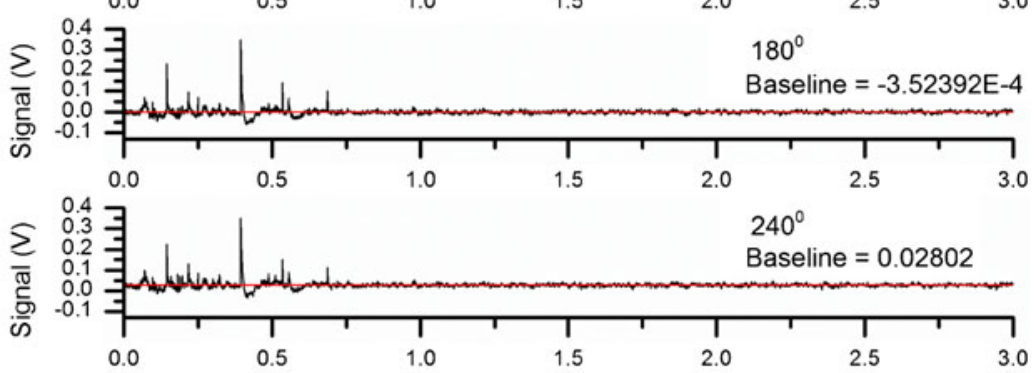

(g)

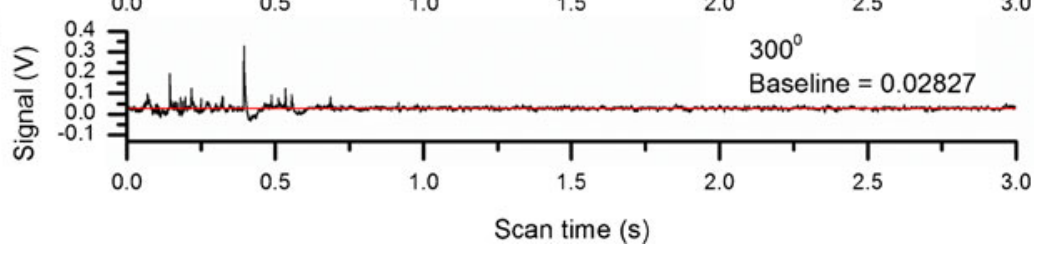

Figure 2. Signal resampling at different if driving phases. (a) The signal after boxcar averaging and harmonic cancellation. (b) to (g) The resampling signals and those baseline fittings are at the phase of $0,60,120,180,240$, and 300 degrees

Now we return to how to remove the rf interference in signals. We decompose each row of $\mathrm{S}[\mathrm{m}, \mathrm{n}]$ by OWPD until $\mathrm{J}=2$ of $d_{j}^{p}$ in Equation (B.3) (shown in supporting information B) for baseline estimation. The decomposition coefficients are $d[m, n]_{2}^{0}, d[m, n]_{2}^{1}, d[m, n]_{2}^{2}$, and $d[m, n]_{2}^{3}$. The scaling function $\mathrm{g}[\mathrm{n}]$ is a low pass filter and $d[m, n]_{2}^{0}$ is operated by $\mathrm{g}[\mathrm{n}]$ with two times. We refer $d[m, n]_{2}^{0}$ to an approximated coefficient and estimate the baseline $\mathrm{b}[\mathrm{m}]$ in $d[m, n]_{2}^{0}$ by fitting with a horizontal linear line, and then remove the nonzero baseline back to zero as

$$
d[m, n]_{2}^{0 \dagger}=d[m, n]_{2}^{0}-b[m]
$$

Now we reconstruct the $\mathrm{S}[\mathrm{m}, \mathrm{n}]$ by using the new decomposition coefficient $d[m, n]_{2}^{0} \dagger$ and all other three decomposition coefficients $d[m, n]_{2}^{1}, d[m, n]_{2}^{2}$, and $d[m, n]_{2}^{3}$. Finally, $\mathrm{S}[\mathrm{m}, \mathrm{n}]$ is rearranged to a one-dimensional array by using time stamp $\mathrm{t}_{\mathrm{rs}}[\mathrm{m}, \mathrm{n}]$ arranged from low to high.

\section{Results and Discussion}

\section{Mass Calibration of a LDCD-ITMS}

When a particle is ejected axially from QIT in the monopolar frequency-scan mode, its $m / Z e$ value can be calculated according to

$$
\frac{m}{Z e}=K \frac{V_{\text {eject }}}{\Omega_{\text {eject }}{ }^{2}}
$$

where $\mathrm{m}, \mathrm{Z}$, and e are the particle mass, the charge number, and the elementary charge; $\mathrm{V}_{\text {eject }}$ and $\Omega_{\text {eject }}$ are the amplitude and the frequency of driving voltage of QIT at the particle ejection point; $\mathrm{K}$ is a static coefficient.

The output signal $\mathrm{V}_{\mathrm{CD}}$ of charge detector is linearly proportional with charge number

$$
Z e=k V_{C D}
$$

where $\mathrm{k}$ is a coefficient which is equal to $56.8 \frac{\mathrm{ke}}{\text { Volt }}$ by the linear regression of signals and the induced charge by test 
(a)

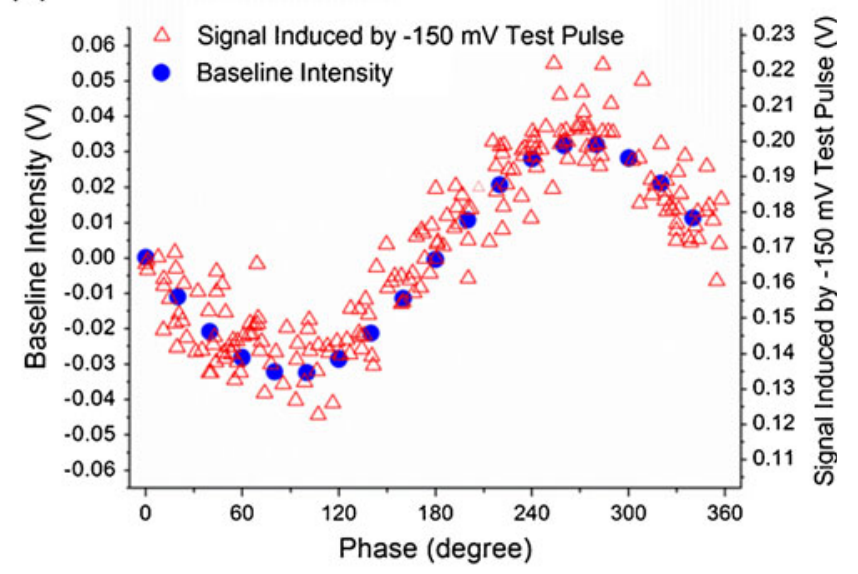

(b)

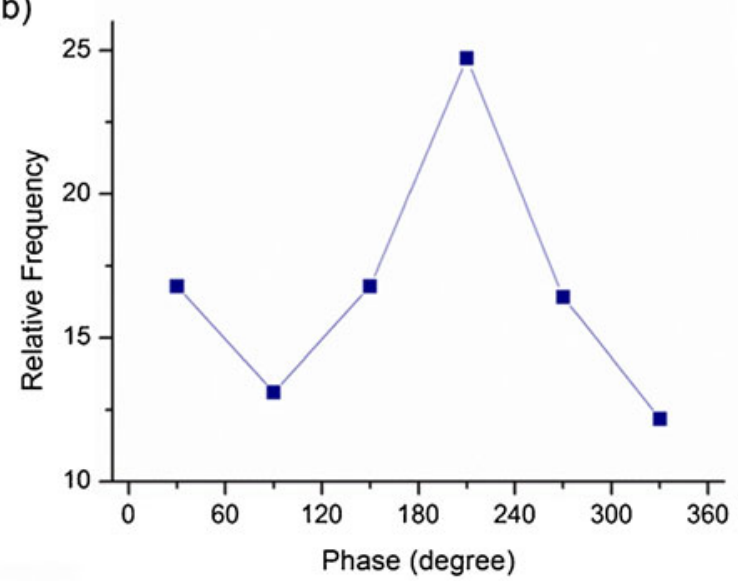

Figure 3. (a) The blue spots are the baseline intensities and the red hollow triangles are the peak heights induced by $150 \mathrm{mV}$ test pulse where the frequency scan condition is set at 5000 to $500 \mathrm{~Hz}$ with a constant driving voltage $850 \mathrm{~V}_{0-p}$. The if driving voltage causes a same distortion in both baseline and peak height, and the intensities of distortion are correlated to different phases of if driving voltage. (b) The population of ejected particles is correlated to different phases of the applied if driving voltage. About $25 \%$ particles are ejected at $\sim 200^{\circ}$

pulses. The coefficient of determination (R-square) of linear regression is 1.00 .

The mass of particle can be calculated by doing multiplication of Equation (12) and Equation (13) as

$$
m=K k \frac{V_{\text {eject }} V_{C D}}{\Omega_{\text {eject }}{ }^{2}}
$$

By removing the constants in Equation (14), we define a 'mass coefficient', which is proportional to the mass of particle:

$$
\text { mass coefficient }=\frac{V_{\text {eject }} V_{C D}}{\Omega_{\text {eject }}{ }^{2}}
$$

The histograms of mass coefficient of different sizes polystyrene are shown in supporting information E. Table shown in supporting information $\mathrm{F}$ is the mass calibration table where the mean mass coefficients are obtained by Gauss fitting. The theoretic masses are then calculated by the given volumes and density of size standard polystyrene particles. The R-square obtained by linear regression is 1.0. This demonstrates LDCD-ITMS shows high linearity and accuracy between mass and mass coefficient ranging from $2.14 \times 10^{12}$ to $2.64 \times 10^{15} \mathrm{Da}$. To get the mass distribution of an unknown analyte, we measure the mass coefficient of analyte by LDCD-ITMS and then do the linear interpolation of calibration curve with the known mass coefficient.

\section{Noise Reduction and Its Correlation to Mass Resolution}

Shown in Figure 4a and Figure $4 \mathrm{~b}$ are the real signal and denoised signal of a $0.7 \mu \mathrm{m}$ polystyrene size standard. It is obvious that more peaks can be distinguished after denoising. In Figure $4 a$, rf interference is clearly observed and causes significant distortion. The reason for this distortion is that the trapping frequency of QIT is higher than the response speed of detector. The $\mathrm{S} / \mathrm{N}$ ratios of peak 1 and peak 2 in Figure 4a are 6.52 and 4.80. Insert in Figure 4a shows the distorted peaks are superimposed to real peak shape. Shown in Figure $4 b$, we notice the $\mathrm{S} / \mathrm{N}$ ratios of peak 1 and peak 2 are greatly improved to 21.7 and 15.4 after denoising. The OWPD based $\mathrm{rf}$ cancellation algorithm successfully removes the distortion. We calculate the rootmean-square values of those noises, and the noise background is equal to $24.1 \mathrm{mV}$ (1366 electrons). After denoising, the noise background is reduced to $7.2 \mathrm{mV}$ (408 electrons). The noise components in Figure $4 \mathrm{~b}$ are very close to white noise. To sum up, in LDCD-ITMS, the noise is reduced $70 \%$ off by denoising program and the detection limit (a signal greater than three times the standard deviation of the noise level) is improved from $72.3 \mathrm{mV}$ (4100 electrons) to $21.6 \mathrm{mV}$ (1225 electrons). When trapping nanoparticles, the trapping frequency should be tuned higher than $1 \mathrm{kHz}$. In this condition, the $\mathrm{S} / \mathrm{N}$ ratios will be greatly improved by removing the superimposed rf noises in real signals.

To discuss the noise components of the charge detector, we use the test pulse to measure the response signals. The noise intensity of $\mathrm{N}_{\mathrm{RF}}$ we obtained is about $32 \mathrm{mV}\left(\mathrm{V}_{0-\mathrm{p}}\right)$ which is converted to $22.6 \mathrm{mV}_{\text {rms }}$ (1283 electrons) in rootmean-square value as shown in Figure 4a. In our previous study, the electronic noise of charge detector is about 500 electrons while the driving voltage of QIT is turned off [24]. This means that the noise components $\left(\mathrm{N}_{\mathrm{h}}\right.$ and $\left.\mathrm{N}_{\mathrm{w}}\right)$ of the charge detector is 500 electrons. The error propagation of LDCD-ITMS can be expressed as [32]:

$$
\sigma\left(N_{\text {total }}\right)^{2}=\sigma\left(N_{R F}\right)^{2}+\sigma\left(N_{h}\right)^{2}+\sigma\left(N_{w}\right)^{2}
$$



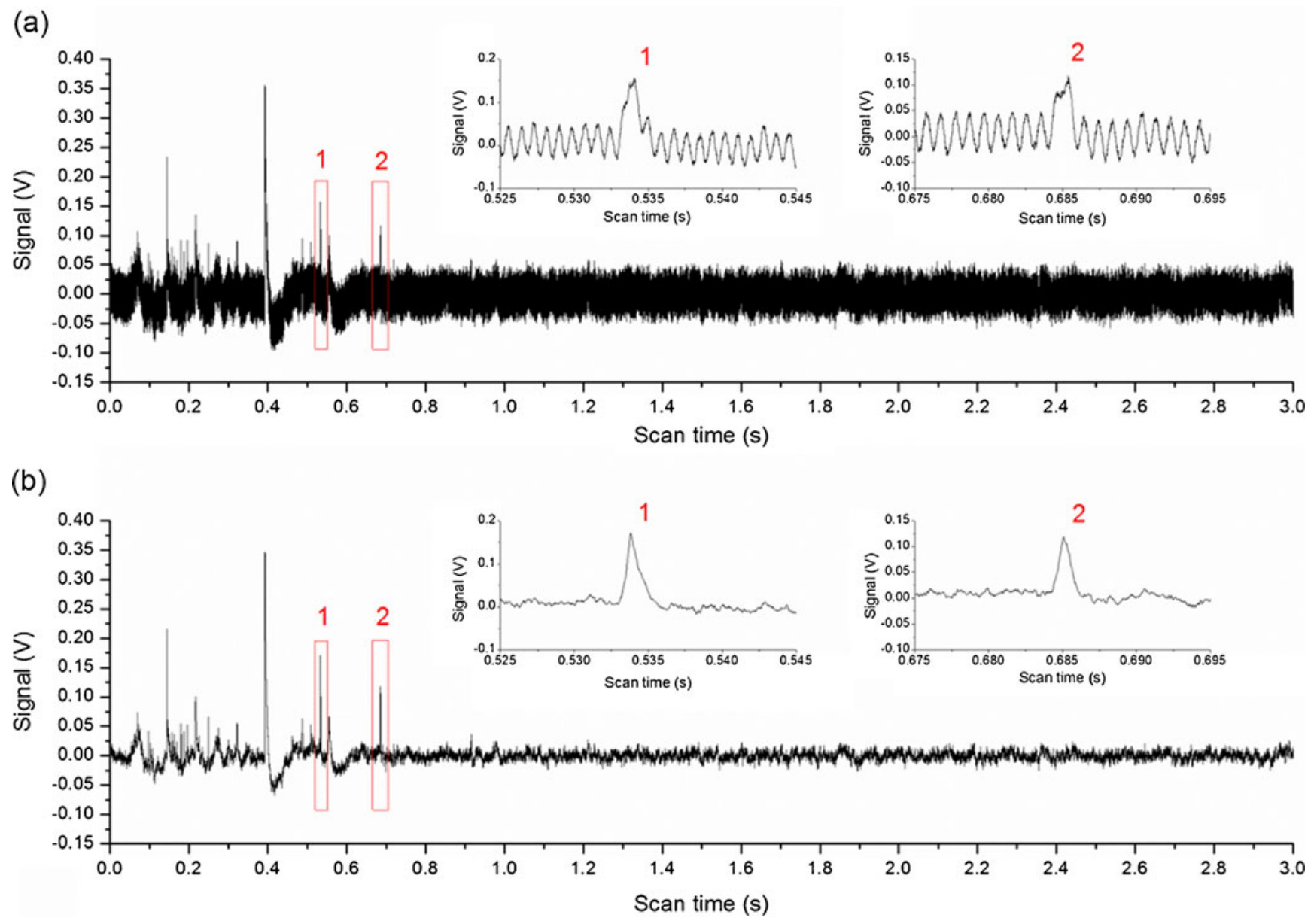

Figure 4. Noise reduction by the denoising program. (a) Frequency scan raw spectrum of $0.7 \mu \mathrm{m}$ diameter polystyrene. The trapping frequency $\left(5000 \mathrm{~Hz}, 850 \mathrm{~V}_{0-p-p}\right.$ ) for $0.7 \mu \mathrm{m}$ diameter polystyrene particle is higher than the response frequency of the charge detector. The rf and harmonic interferences from QIT causes a significant distortion. (b) Denoised mass spectrum of (a). The denoising program removes all the distortions in the mass spectrum
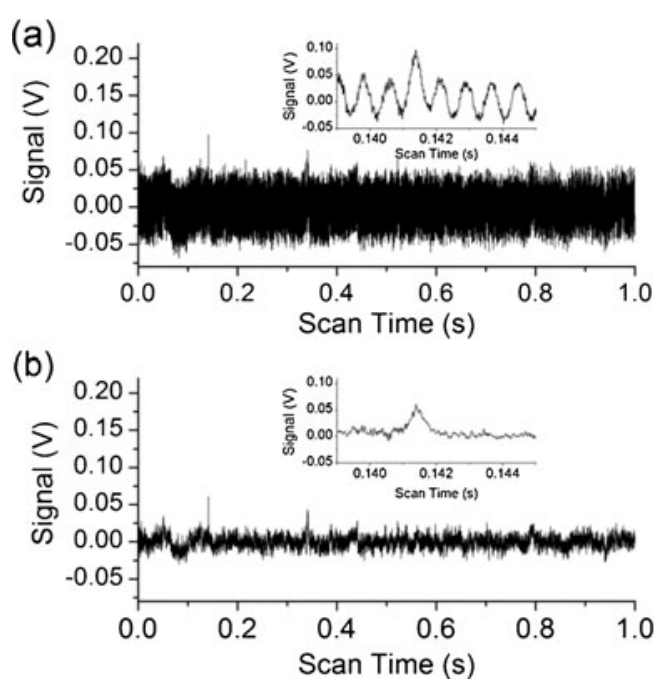

Figure 5. (a) Frequency scan raw spectrum $(5050$ to $500 \mathrm{~Hz}$, $850 \mathrm{~V}_{p-p}$ ) of Escherichia coli without denoising. (b) Denoised spectrum of (a) where $\sigma\left(\mathrm{N}_{\mathrm{RF}}\right), \sigma\left(\mathrm{N}_{\mathrm{h}}\right)$, and $\sigma\left(\mathrm{N}_{\mathrm{w}}\right)$ are the standard deviations of the rf interference, the harmonic interference and the white noise, respectively. According to Equation (16), we have that $\sqrt{\sigma\left(N_{h}\right)^{2}+\sigma\left(N_{w}\right)^{2}}$ is 500 electrons and $\sigma\left(\mathrm{N}_{\mathrm{RF}}\right)$ is 1283 electrons, therefore the $\sigma\left(\mathrm{N}_{\text {total }}\right)$ is 1377 electrons. The $\sigma\left(\mathrm{N}_{\text {total }}\right)$ is in consistent with the noise background (1367 electrons) in our experiment (Figure 4a).

It is difficult for bacteria detection using charge detector by thresholding peak detection method without $\mathrm{N}_{\mathrm{RF}}$ cancellation. The charge number of bacteria is typically less than 4200 ( $\sim 3$ times of noise background). Figure 5a shows a frequency scan mass spectrum of Escherichia coli. Without denoising, only one distorted peak can be identified. After denoising, as shown in Figure 5b, two peaks can be clearly identified. We therefore can estimate the peak height without the distortion induced by $\mathrm{N}_{\mathrm{RF}}$ and $\mathrm{N}_{\mathrm{h}}$.

Figure $6 \mathrm{a}$ and $\mathrm{b}$ show the histograms of mass coefficient of $4 \mu \mathrm{m}$ size standard polystyrene. Figure $6 \mathrm{a}$ is plotted with the peaks above $130 \mathrm{mV}$ and Figure $6 \mathrm{~b}$ is 
(a)

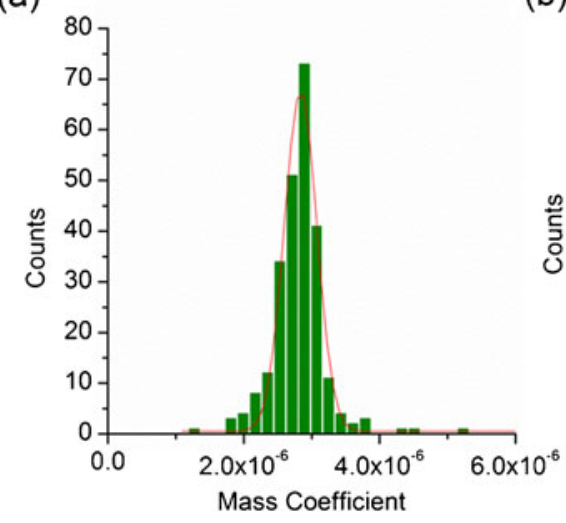

(b)

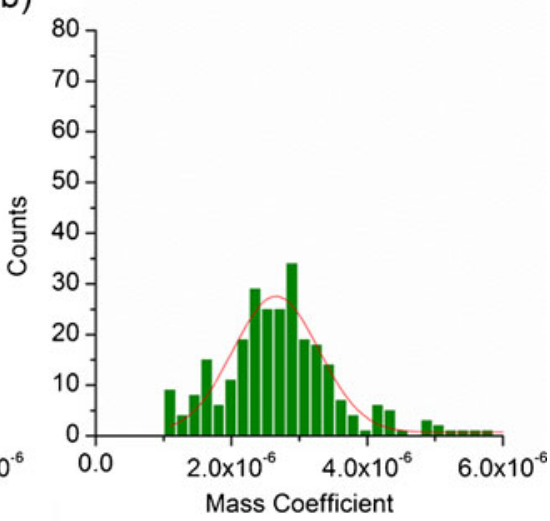

(c)

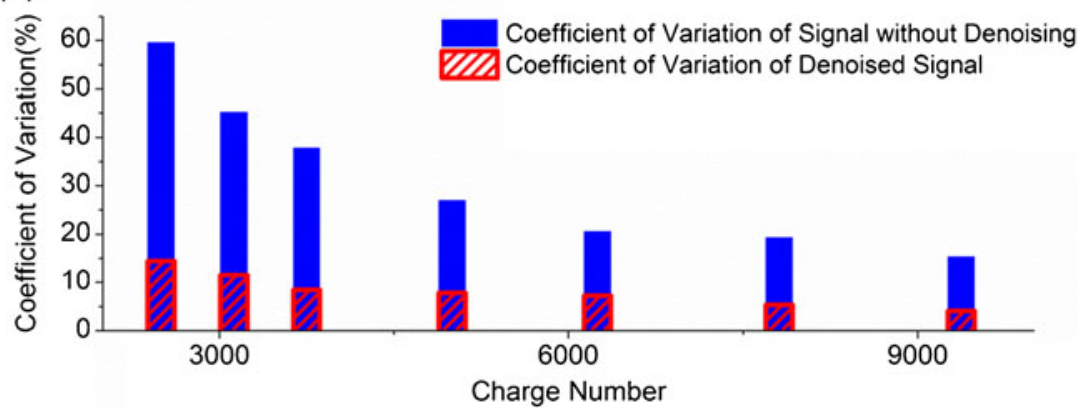

Figure 6. (a) Mass coefficient histograms of different peaks with a threshold above $130 \mathrm{mV}$ and (b) below $130 \mathrm{mV}$ of $4 \mu \mathrm{m}$ diameter polystyrene particles. These two diagrams show that a better mass resolution could be obtained for particles with higher charges. (c) Coefficients of variation of measured charges induced by test pulse

plotted with the peaks below $130 \mathrm{mV}$. The coefficients of variation $(\mathrm{CV})$ are $8.45 \%$ and $23.69 \%$ in Figure $6 \mathrm{a}$ and Figure $6 \mathrm{~b}$, respectively. This means that the resolution of LDCD-ITMS is strongly correlated with the charge carried on the analyte.

Now we examine the imprecision of LDCD-ITMS in detail. The imprecision is from two major sources: (1) imprecision of the mass analyzer, (2) imprecision of the charge detector. We discuss the imprecision of the mass analyzer in two parts. The first part is the effect of driving voltage of QIT. Nie et al. showed that a higher resolution of QIT can be obtained if we set higher driving voltage [26]. This explains why the resolution in high mass analysis is not good when the QIT is applied with lower driving voltage under frequency scan mode. The second part might be the limited step number in a frequency scan. The function generator uses a phase-continuous frequency hopping mode for frequency scan. As shown in Equation (12), the number of steps means how many $m / Z e$ values can be distinguished by stepwise frequency scan. A possible guess is that the mass resolution is poor at high frequency side. Nie et al. showed the resolution of $m / Z e$ is $\sim 100$ for a single particle analysis by frequency scan using function generator SRS-345, which has 128 steps for stepwise frequency scan [33]. In this study, we adopt 1000 steps for stepwise frequency scan to analyze multiple particles ejected out of the ion trap. As shown in Figure 6a, the best resolution of mass measurement is 11.8; therefore, the imprecision is caused by charge detection part. To estimate the imprecision of the charge detector, we had to find the relationship between the charge number and the $\mathrm{CV}$ of a charge detector signal induced by test pulse. The result is shown in Figure 6c. The diagram clearly shows that the denoising program is essential to enhance the mass precision and improve the detection limit. The remained impression of charge detector after denoising is about 400 electrons, which is pure white noise. However, before we reduce the noise level of a charge detector, it is very hard to estimate the real mass precision of $m / Z e$ of multiple particles trapped by LDCD-ITMS using frequency scan mode.

After analysis of all noise components and characteristics of LDCD-ITMS, white noise is the only component to be overcome. To improve the detection limit and precision of the charge detector, electronic white noise is crucial to improve the performance of detecting nanometer-sized particles. Redesigning the electronic circuit of charge detector with a lower input noise junction field effect transistor (JFET) coupled with a cooling stage might be a practical solution to improve the performance of the charge detector [34]. Therefore direct detection of nanoparticles using LDCD-ITMS might become possible. 


\section{Conclusions}

We have developed a denoising program based on an OWPD method to effectively remove the interference induced by the applied rf driving voltage, which is inevitably present in LDCD-ITMS. The program shows high efficacy in eliminating the rf-induced noise, removing the harmonics from the $\mathrm{AC}$ power source, and reducing the white noise level by Boxcar averaging with wavelet decomposition coefficient threshold. Tested with the induced rf signals from the charge detector, the method successfully reduces the noise level in charge detection from 1367 to 408 electrons and improves the detection sensitivity limit by about $70 \%$. The denoising program will help charge detection devices to operate in strong rf field without the rf interferences [35-38] and is expected to be useful to remove the interferences from the applied high rf voltages used in various types of mass spectrometers, including quadrupole ion trap, linear ion trap, Fourier transform ion cyclotron resonance, and Orbitrap mass spectrometers [39, 40].

\section{Acknowledgment}

The authors thank the National Science Council (grant 992112-M-259-002-MY3) of Taiwan for financial support (W.P.P.)

\section{References}

1. Cappadona, S., Levander, F., Jansson, M., James, P., Cerutti, S., Pattini, L.: Wavelet-based method for noise characterization and rejection in high-performance liquid chromatography coupled to mass spectrometry. Anal. Chem. 80, 4960-4968 (2008)

2. Morris, J.S., Coombes, K.R., Koomen, J., Baggerly, K.A., Kobayashi, R.: Feature extraction and quantification for mass spectrometry in biomedical applications using the mean spectrum. Bioinformatics 21, 1764-1775 (2005)

3. Coombes, K.R., Tsavachidis, S., Morris, J.S., Baggerly, K.A., Hung, M.C., Kuerer, H.M.: Improved peak detection and quantification of mass spectrometry data acquired from surface-enhanced laser desorption and ionization by denoising spectra with the undecimated discrete wavelet transform. Proteomics 5, 4107-4117 (2005)

4. Wang, P.H., Yang, P.Y., Arthur, J., Yang, J.Y.H.: A dynamic waveletbased algorithm for pre-processing tandem mass spectrometry data. Bioinformatics 26, 2242-2249 (2010)

5. Du, P., Kibbe, W.A., Lin, S.M.: Improved peak detection in mass spectrum by incorporating continuous wavelet transform-based pattern matching. Bioinformatics 22, 2059-2065 (2006)

6. Li, X.L., Li, J., Yao, X.: A wavelet-based data pre-processing analysis approach in mass spectrometry. Comput. Biol. Med. 37, 509-516 (2007)

7. Andreev, V.P., Rejtar, T., Chen, H.S., Moskovets, E.V., Ivanov, A.R., Karger, B.L.: A universal denoising and peak picking algorithm for LCMS based on matched filtration in the chromatographic time domain. Anal. Chem. 75, 6314-6326 (2003)

8. Erickson, C.L., Lysaght, M.J., Callis, J.B.: Relationship between digital filtering and multivariate regression in quantitative analysis. Anal. Chem. 64, 1155A-1163A (1992)

9. Barclay, V.J., Bonner, R.F., Hamilton, I.P.: Application of wavelet transforms to experimental spectra: Smoothing, denoising, and data set compression. Anal. Chem. 69, 78-90 (1997)

10. Oppenheim, A.V., Willsky, A.S., Young, I.T.: Signals and systems. Prentice-Hall, (1983)

11. Xu, L.J.: Cancellation of harmonic interference by baseline shifting of wavelet packet decomposition coefficients. IEEE T Sig. Proces. 53, 222-230 (2005)
12. Xu, L.J., Yan, Y.: Wavelet-based removal of sinusoidal interference from a signal. Meas. Sci. Technol. 15, 1779-1786 (2004)

13. Liguori, C., Paolillo, A., Pignotti, A.: Estimation of signal parameters in the frequency domain in the presence of harmonic interference: A comparative analysis. IEEE T Instrum. Meas. 55, 562-569 (2006)

14. Douglas A. Skoog, F.J.H., Timothy A. Nieman. Principles of Instrumental Analysis. Thomson Learning, (1998)

15. Delmas, J.P.: Adaptive harmonic jammer canceler. Sig. Process. IEEE T. 43, 2323-2331 (1995)

16. Fainzilberg, L.S., Glushauskene, G.A.: Narrow-band rejection filter for suppression of harmonic concentrated interference on the basis of discrete fourier transform. J Automat. Inf. Sci. 41, 5570 (2009)

17. Tsai, C.D., Chiou, D.C., Lin, Y.D., Chan, H.L., Wu, C.P.: An active comb filter design for harmonic interference removal. J. Chin. Inst. Eng. 21, 605-610 (1998)

18. Zhang, S., Li, D., Sun, J.: Power system frequency tracking using an adaptive lattice notch filter. Key Eng. Mat. 295/296, 673-678 (2005)

19. Chen, B.-S., Yang, T.-Y., Lin, B.-H.: Adaptive notch filter by direct frequency estimation. Sig. Process. 27, 161-176 (1992)

20. Glover Jr., J.: Adaptive noise canceling applied to sinusoidal interferences. Acoustics, Speech, and Signal Processing. IEEE T. 25, 484-491 (1977)

21. Pilt, K., Meigas, K., Ferenets, R., Kaik, J.: Adjustment of adaptive sum comb filter for PPG signals. Proceedings of the IEEE Conference. Eng. Med. Biol. Soc. 2009, 5693-5696 (2009)

22. Nie, Z.X., Lin, C.W., Peng, W.P., Lee, Y.T., Chang, H.C.: Frequency scan of a quadrupole mass analyzer in the third stability region for protein analysis. J. Chin. Chem. Soc. 53, 47-52 (2006)

23. Peng, W.P., Lin, H.C., Chu, M.L., Chang, H.C., Lin, N.H., Yu, A.L., Chen, C.H.: Charge monitoring cell mass spectrometry. Anal. Chem. 80, 2524-2530 (2008)

24. Peng, W.P., Lin, H.C., Lin, H.H., Chu, M., Yu, A.L., Chang, H.C., Chen, C.H.: Charge-monitoring laser-induced acoustic desorption mass spectrometry for cell and microparticle mass distribution measurement. Angew. Chem. Int. Ed. 46, 3865-3869 (2007)

25. Peng, W.P., Yang, Y.C., Kang, M.W., Tzeng, Y.K., Nie, Z.X., Chang, H.C., Chang, W., Chen, C.H.: Laser-induced acoustic desorption mass spectrometry of single bioparticles. Angew. Chem. Int. Ed. 45, 14231426 (2006)

26. Xiong, C., Xu, G., Zhou, X., Wang, J., Tang, Y., Chen, R., Peng, W.P., Chang, H.C., Nie, Z.: The development of charge-detection quadrupole ion trap mass spectrometry driven by rectangular and triangular waves. Analyst 137, 1199-204 (2012)

27. Xiong, C.Q., Zhou, X.Y., Chen, R., Zhang, Y.M., Peng, W.P., Nie, Z.X., Chang, H.C., Liu, H.W., Chen, Y.: Characterization of column packing materials in high-performance liquid chromatography by charge-detection quadrupole ion trap mass spectrometry. Anal. Chem. 83, 5400-5406 (2011)

28. Peng, W.P., Yang, Y.C., Lin, C.W., Chang, H.C.: Molar mass and molar mass distribution of polystyrene particle size standards. Anal. Chem. 77, 7084-7089 (2005)

29. Lin, H.C., Lin, H.H., Kao, C.Y., Yu, A.L., Peng, W.P., Chen, C.H.: Quantitative measurement of nano-/microparticle endocytosis by cell mass spectrometry. Angew. Chem. Int. Ed. 49, 3460-3464 (2010)

30. Schlunegger, U.P., Stoeckli, M., Caprioli, R.M.: Frequency scan for the analysis of high mass ions generated by matrix-assisted laser desorption/ionization in a Paul trap. Rapid Commun. Mass Spectrom. 13, 1792-1796 (1999)

31. Mallat, S.: A Wavelet Tour of Signal Processing, 3rd ed: The Sparse Way. Academic Press, (2008)

32. Taylor, J.R.: An introduction to error analysis: The study of uncertainties in physical measurements. University Science Books, Mill Valley, California (1982)

33. Nie, Z., Cui, F., Chu, M., Chen, C.H., Chang, H.C., Cai, Y.: Calibration of a frequency-scan quadrupole ion trap mass spectrometer for microparticle mass analysis. Int. J. Mass spectrom. 270, 8-15 (2008)

34. See Amptek A250CF in http://www.amptek.com. Accessed March 01 2012

35. Benner, W.H.: A gated electrostatic ion trap to repetitiously measure the charge and $m / z$ of large electrospray ions. Anal. Chem. 69, 4162-4168 (1997) 
36. Benner, W.H., Bogan, M.J., Rohner, U., Boutet, S., Woods, B., Frank, M.: Non-destructive characterization and alignment of aerodynamically focused particle beams using single particle charge detection. J. Aerosol Sci. 39, 917-928 (2008)

37. Fuerstenau, S.D., Benner, W.H.: Molecular weight determination of megadalton DNA electrospray ions using charge detection time-offlight mass spectrometry. Rapid Commun. Mass Spectrom. 9, 15281538 (1995)
38. Smith, J.W., Siegel, E.E., Maze, J.T., Jarrold, M.F.: Image charge detection mass spectrometry: Pushing the envelope with sensitivity and accuracy. Anal. Chem. 83, 950-956 (2011)

39. Reilly, J.P.: Neuroelectric mechanisms applied to low frequency electric and magnetic field exposure guidelines - Part I: Sinusoidal waveforms. Health Phys. 83, 341-355 (2002)

40. Hu, Q.Z., Noll, R.J., Li, H.Y., Makarov, A., Hardman, M., Cooks, R.G.: The Orbitrap: A new mass spectrometer. J. Mass Spectrom. 40, 430-443 (2005) 